\title{
Study of Diastolic Function in Asymptomatic Type 2 Diabetes Patients Visiting Goa Medical College and Hospital, Bambolim, Goa
}

\author{
Vinay Kumar A.V.1 , Sumeet Juwarkar², Viraj Khandeparkar³ \\ 1,2,3 Department of Medicine, Goa Medical College, Bambolim, Goa. India.
}

\section{ABSTRACT}

\section{BACKGROUND}

Diabetes mellitus increases the risk of heart failure even in the absence of other comorbidities. The present study is done to assess the diastolic dysfunction in diabetic patients and in correlating the grade of dysfunction with various parameters like age, gender, sex, glycaemic control, and microangiopathies associated with diabetes. We wanted to determine as to whether there is an association between diastolic dysfunction and type $2 \mathrm{DM}$ and quantify the relation of LV diastolic dysfunction with age, duration of $\mathrm{DM}, \mathrm{HbA} 1 \mathrm{c}$ and obesity indices. We also wanted to assess the correlation between diastolic dysfunction and other microvascular complications of type 2 diabetes.

\section{METHODS}

This is a cross sectional study. The sample size was 75. Assessment of the mitral peak velocity of early filling (E) and early diastolic mitral annular velocity ( $\left.e^{\prime}\right)$, and ( $E$ / e') ratio is used to measure diastolic dysfunction. study was conducted on patients admitted in Goa Medical College.

\section{RESULTS}

Among 75 subjects studied, 56 subjects (74.6 \%) had diastolic dysfunction. There is a correlation of duration of diabetes with grade of diastolic dysfunction with a p value of 0.001 . There is a correlation of dipstick proteinuria with grades of diastolic dysfunction with a $\mathrm{p}$ value of 0.002 . Mean $\mathrm{HbA1c}$ in patients with grade 0 dysfunction was 7.93 with standard deviation of 0.86 , grade 1 dysfunction was 7.28 with a standard deviation of 0.71 , grade 2 dysfunction was 9.72 with standard deviation of 1.86 , grade 3 dysfunction was 11.94 with standard deviation of 2.05 with a $p$ value of $<0.001$.

\section{CONCLUSIONS}

Diabetes is an independent risk factor for cardiovascular disease in the form of diastolic dysfunction in the initial stages. The grade of dysfunction was proportional to duration of diabetes, blood sugar levels, and mean HbA1c. Diastolic dysfunction also correlates with other microvascular complications of diabetes.

\section{KEY WORDS}

Diastolic Dysfunction, Diastolic Function in Diabetes
Corresponding Author: Dr. Vinay Kumar A.V. Department of Medicine, Goa Medical College, Bambolim, Goa. E-mail: vinaykumarav91@gmail.com

DOI: $10.14260 / \mathrm{jemds} / 2020 / 707$

How to Cite This Article:

Vinay Kumar AV, Juwarkar S, Khandeparkar V. Study of diastolic function in asymptomatic type 2 diabetes patients visiting Goa medical college and hospital, Bambolim, Goa. J Evolution Med Dent Sci 2020;9(43):3223-3226, $10.14260 /$ jemds/2020/707

Submission 18-07-2020,

Peer Review 16-09-2020

Acceptance 23-09-2020,

Published 26-10-2020.

Copyright (C) 2020 Vinay Kumar A.V. et al. This is an open access article distributed under Creative Commons Attribution License [Attribution 4.0 International (CC BY 4.0)] 


\section{BACKGROUND}

The number of cases of diabetes mellitus worldwide in 2000 among adults of more than 20 years of age is estimated to be 171 million. ${ }^{1}$ The existence of a diabetic cardiomyopathy has been proposed as evidence strongly pointing towards presence of myocardial dysfunction in diabetic patients in the absence of hypertensive, valvular or ischemic heart disease. Diabetes mellitus increases the risk of heart failure even in the absence of other co-morbidities. Diastolic dysfunction is an inability of the ventricle to fill to a normal end-diastolic volume, both during exercise as well as at rest.

Diabetic cardiomyopathy is defined as the presence of myocardial involvement in patients with diabetes, characterized by dilatation and hypertrophy of the left ventricle, with the concomitant appearance of diastolic and / or systolic dysfunction, and its presence is independent of the coexistence of ischemic or hypertensive or valvular heart disease. $^{2}$

The present study is done to assess diastolic dysfunction in diabetic patients and in correlating the grade of dysfunction with various parameters like age, gender, sex, glycaemic control and microangiopathies associated with diabetes.

Nichols GA et $\mathrm{al}^{3}$ conducted a cohort study on 8231 patients with type 2 diabetes and 8845 nondiabetic patients of similar age and sex for about 72 months and found out that patients with diabetes were much more likely to develop congestive heart failure than patients without diabetes.

The mechanism of diabetic cardiomyopathy is multifactorial including myocyte dysfunction, caused by metabolic disturbances, myocardial fibrosis, autonomic dysfunction, microvascular diseases and insulin resistance. ${ }^{4}$ Myocardial fibrosis, as initially described by Rubler et al and confirmed in histological studies in both experimental subjects and humans, is a major consequence of the adverse effects of diabetes mellitus in the heart.

Microalbuminuria is considered the marker for renal microangiopathy. Degree of albuminuria correlates with severity of microangiopathy. Microangiopathy progressing to myocardial dysfunction is gaining attention recently. The exact relation between microalbuminuria and cardiomyopathy remains elusive.

At present, echocardiography is the best way to evaluate diastolic function and in estimating filling pressures.

Assessment of diastolic function by echocardiography [European Association of Echocardiography (EAE)].

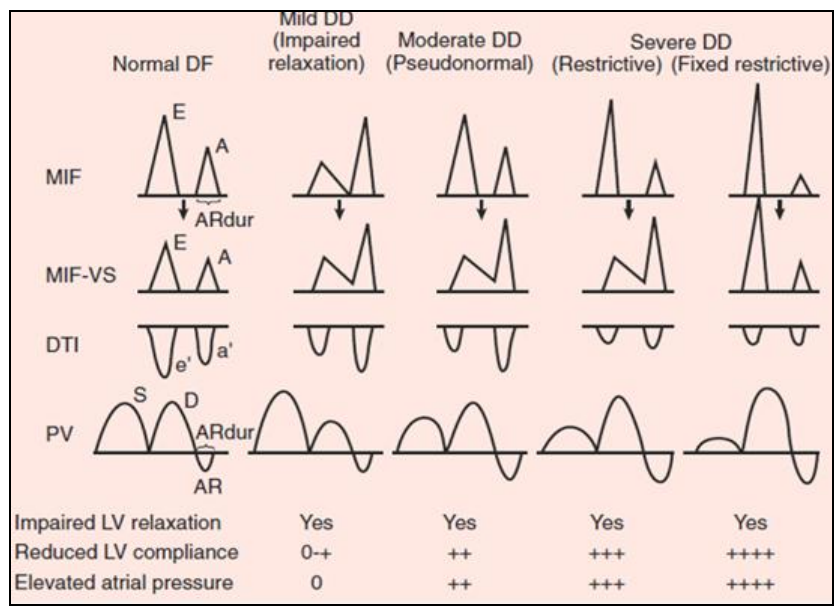

[E, peak early filling velocity; A, velocity at atrial contraction; DT, deceleration time; Adur, Atrial contraction duration; ARdur, Atrial relaxation duration; S, systolic forward flow; D, diastolic forward flow; AR, pulmonary venous atrial reversal flow; e', velocity of mitral annulus early diastolic motion; a', velocity of mitral annulus motion with atrial systole; DT, mitral E velocity deceleration time.]

(Modified from Redfield MM, Jacobsen SJ, Burnett JC Jr, et al: Burden of systolic and diastolic ventricular dysfunction in the community. Appreciating the scope of the heart failure epidemic. JAMA 289:194, 2003). ${ }^{5}$

\section{METHODS}

This is a cross sectional study conducted among patients admitted in Goa Medical College over a period of two years from september 2016 to august 2018. The sample size was 75 . Sample size was chosen arbitrarily. Assessment of the mitral peak velocity of early filling, early diastolic mitral annular velocity and E / e' ratio were done. Echocardiography was done by a single trained cardiologist. Grades of diastolic dysfunction were derived as per European Association of Echocardiography guidelines for diastolic dysfunction. Approval was taken from information and Ethical Committee of Goa Medical College on 14/10/16.

\section{Inclusion Criteria}

- TYPE 2 DM was diagnosed according to American Diabetes Association criteria. ${ }^{6}$

- All type 2 DM patients with duration > 3 years with normal left ventricular systolic function [LVEF > $50 \%$ ].

- Patients free of cardio vascular complaints.

- Normal ECG without rhythm disturbances.

\section{Exclusion Criteria}

- Patients with angina or anginal equivalent symptoms (dyspnoea, nausea, fatigue and faintness, abdominal pain.)

- Hypertensive patients. [According to JNC 7 blood pressure classification system.]

- $\quad$ ECG changes suggestive of IHD.

- Abnormal treadmill test rest results (if available).

- Evidence of valvular heart diseases.

\section{Stastical Analysis}

Statistical analysis was conducted using SPSS 10.0 software package. Statistical significance was determined using chi square test and Anova test for covariance and $p<0.05$ was considered significant.

\section{RESULTS}

A total of 75 patients both males and females was studied during the period of 18 months. Youngest was 36 years old and oldest subject was 80 years old. Out of 75 subjects 47 subjects were males and 28 subjects were females. Among 12 patients 
who had diabetes for atleast 7 to 10 years, 2 patients (16.7\%) had grade 0 dysfunction, 1 patient $(8.3 \%)$ had grade 1 dysfunction, 8 patients $(66.7 \%)$ had grade 2 diastolic dysfunction, 1 patient $(8.3 \%)$ had grade 3 diastolic dysfunction. Among 4 patients who had diabetes for atleast 11 to 15 years 1 patient ( $25 \%$ ) had grade 2 dysfunction, 3 patients (75\%) had grade 3 dysfunction. Among 3 patients who had diabetes for more than 15 years, 1 patient (33\%) had grade 2 dysfunction, 2 patients (66 \%) had grade 3 dysfunction. Chi square test applied for correlation of duration of diabetes with diastolic dysfunction showed $\mathrm{p}$ value of < 0.001. Among 7 patients with no diabetic retinopathy, 5 patients $(71.4 \%)$ had grade 0 diastolic dysfunction, 2 patients (28.6\%) had grade 1 dysfunction. Among 31 patients with mild NPDR 13 patients (41.9\%) had grade 0 dysfunction, 5 patients (16.1\%) had grade 1 dysfunction, 12 patients $(38.7$ $\%$ ) had grade 2 dysfunction, 1 patients (3.2\%) had grade 3 dysfunction. Among 29 patients with moderate NPDR 1 patient (3.4\%) had grade 0 dysfunction, 1 patient (3.4\%) had grade 1 dysfunction, 24 patients $(82.8 \%)$ had grade 3 dysfunction, 3 patients $(10.3 \%)$ had grade 4 dysfunction Among 8 patients who had PDR 5 patients (62.5\%) had grade 2 dysfunction, 3 patients ( $37.5 \%$ ) had grade 3 dysfunction.

\begin{tabular}{|cccccc|}
\hline Duration of & \multicolumn{5}{c|}{ Diastolic Dysfunction } \\
Diabetes & $0(\mathrm{n}=19)$ & $1(\mathrm{n}=8)$ & $2(\mathrm{n}=41)$ & $3(\mathrm{n}=7)$ & \\
(in Years) & $\mathrm{n}(\%)$ & $\mathrm{n}(\%)$ & $\mathrm{n}(\%)$ & $\mathrm{n}(\%)$ & Total \\
3 & $4(40.0)$ & 0 & $6(60.0)$ & 0 & 10 \\
$4-6$ & $13(28.3)$ & $7(15.2)$ & $25(54.3)$ & $1(2.2)$ & 46 \\
$7-10$ & $2(16.7)$ & $1(8.3)$ & $8(66.7)$ & $1(8.3)$ & 12 \\
$11-15$ & 0 & 0 & $1(25.0)$ & $3(75.0)$ & 4 \\
$>15$ & 0 & 0 & $1(33.3)$ & $2(66.7)$ & 3 \\
\hline Association of Duration of Diabetes and Diastolic Dysfunction \\
\hline
\end{tabular}

\begin{tabular}{|cccccc|}
\hline \multirow{7}{*}{ Diastolic Dysfunction Grade } \\
Mild NPDR & $13(41.9)$ & $5(16.1)$ & $12(38.7)$ & $1(3.2)$ & 31 \\
Moderate NPDR & $1(3.4)$ & $1(3.4)$ & $24(82.8)$ & $3(10.3)$ & 29 \\
None & $5(71.4)$ & $2(28.6)$ & 0 & 0 & 7 \\
PDR & 0 & 0 & $5(62.5)$ & $3(37.5)$ & 8 \\
\hline \multicolumn{7}{c}{ Chi-Square Test, P Value $<0.001$, Significant } \\
\hline
\end{tabular}

Among the patients with $1+$ urine albumin 10 (28.6\%) patients had grade 0 diastolic dysfunction, 5 (14.3\%) patients had grade 1 dysfunction, $20(57.1 \%)$ patients had grade 2 dysfunction and $0(0 \%)$ patients had grade 3 dysfunction.

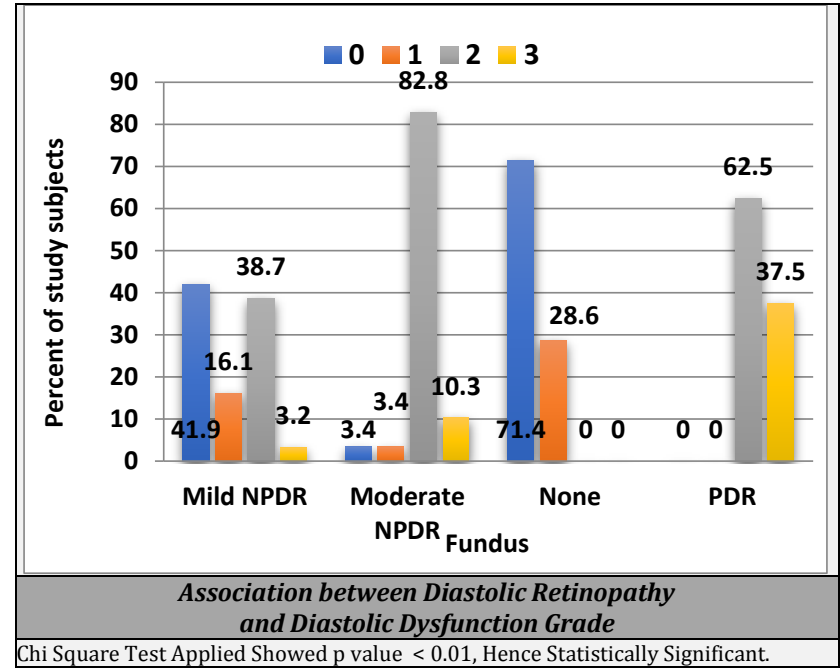

\begin{tabular}{|cccccc|}
\hline Urine & \multicolumn{5}{c|}{ Diastolic Dysfunction grade } \\
Albumin & $0(\mathrm{n}=19)$ & $1(\mathrm{n}=8)$ & $2(\mathrm{n}=41)$ & $3(\mathrm{n}=7)$ & Total \\
& $\mathrm{n}(\%)$ & $\mathrm{n}(\%)$ & $\mathrm{n}(\%)$ & $\mathrm{n}(\%)$ & \\
$1+$ & $10(28.6)$ & $5(14.3)$ & $20(57.1)$ & 0 & 35 \\
$2+$ & $2(11.1)$ & 0 & $11(61.1)$ & $5(27.8)$ & 18 \\
$3+$ & 0 & 0 & 0 & $1(100.0)$ & 1 \\
$4+$ & 0 & 0 & $2(66.7)$ & $1(33.3)$ & 3 \\
Nil & $7(38.9)$ & $3(16.7)$ & $8(44.4)$ & 0 & 18 \\
\hline \multicolumn{7}{|c|}{ Association between Diastolic Dysfunction and Urine Albumin } \\
\hline
\end{tabular}

Among the patients with $4+$ urine albumin $0(0 \%)$ patients had grade 0 dysfunction, $0(0 \%)$ patients had grade 1 dysfunction, $2(66.7 \%)$ had grade 2 dysfunction and 1 (33.3 $\%)$ patient had grade 3 diastolic dysfunction.

Chi square test was applied with a p value of 0.002 which was significant. Post Hoc test applied for duration of diabetes showed statistical significance with $p$ value of $<0.05$ for duration of diabetes, FBS, PPBS, HbA1c with $95 \%$ confidence interval.

\begin{tabular}{|c|c|c|c|c|c|}
\hline \multirow{2}{*}{ Parameter } & \multicolumn{4}{|c|}{ Diastolic Dysfunction [N = 75] } & \multirow[b]{2}{*}{ P Value } \\
\hline & $0(\mathrm{n}=19)$ Mean (SD) & $1(\mathrm{n}=8)$ Mean $(\mathrm{SD})$ & $2(\mathrm{n}=41)$ Mean (SD) & $3(\mathrm{n}=7)$ Mean (SD) & \\
\hline Age (in Years) & $54.47(11.79)$ & $62.87(8.62)$ & $60.00(9.32)$ & $65.29(10.93)$ & 0.054 \\
\hline Duration of Diabetes & $4.63(1.25)$ & $5.38(1.06)$ & $5.90(3.72)$ & $13.29(5.43)$ & $<0.001^{*}$ \\
\hline BMI & $25.63(3.18)$ & $25.25(2.93)$ & $25.86(2.71)$ & $23.91(4.57)$ & 0.473 \\
\hline SBP & $131.16(8.57)$ & $134.75(2.60)$ & $133.37(7.27)$ & $128.86(9.51)$ & 0.329 \\
\hline DBP & $71.58(5.23)$ & $74.25(3.10)$ & $74.34(4.68)$ & $74.00(7.39)$ & 0.250 \\
\hline FBS & $181.84(64.69)$ & $191.75(55.15)$ & $266.02(69.17)$ & $300.71(100.18)$ & $<0.001^{*}$ \\
\hline PPBS & $247.89(74.31)$ & $262.00(89.65)$ & $371.54(98.73)$ & $449.14(93.52)$ & $<0.001^{*}$ \\
\hline $\mathrm{HC}$ & $96.37(4.46)$ & $96.25(3.49)$ & $96.44(3.61)$ & $95.71(3.35)$ & 0.974 \\
\hline WC & $95.68(4.48)$ & $95.25(3.53)$ & $95.59(3.42)$ & $95.14(3.71)$ & 0.984 \\
\hline $\mathrm{HbA1c}$ & $7.93(0.86)$ & $7.28(0.71)$ & $9.72(1.86)$ & $11.94(2.05)$ & $<0.001^{*}$ \\
\hline Blood Urea & $28.74(9.99)$ & $34.13(4.99)$ & $34.07(7.27)$ & $32.86(18.47)$ & 0.219 \\
\hline Serum Creatinine & $0.84(0.15)$ & $0.81(0.06)$ & $0.82(0.20)$ & $0.91(0.27)$ & 0.679 \\
\hline $\mathrm{Hb}$ & $12.06(2.31)$ & $14.12(1.45)$ & $12.70(1.83)$ & $11.52(1.41)$ & $0.037 *$ \\
\hline $\mathrm{TC}$ & 7847 (2275) & 7900 (973) & 7997 (1737) & $9657(3370)$ & 0.209 \\
\hline PCV & $42.47(5.36)$ & $43.50(2.56)$ & $42.95(4.67)$ & $42.71(4.92)$ & 0.961 \\
\hline \multicolumn{6}{|c|}{ ANOVA, p value *Significant. } \\
\hline & Comparison & ns of Variables a & Degree of Diasto & nction & \\
\hline
\end{tabular}

\section{DISCUSSION}

In our study, among 75 subjects studied, 56 subjects (74.6\%) had diastolic dysfunction and 19 (25.3\%) subjects didn't have diastolic dysfunction. This result was similar to study conducted by Kumar VS et $\mathrm{al}^{7}$ in 2017 , Boyer JK et al ${ }^{8}$ who had found $75 \%$ prevalence in 2004, Laura Ponta et $\mathrm{al}^{9}$ conducted study on 62 patients with type 2 diabetes and found significant correlation of diabetes with diastolic dysfunction similar to our study.

There is no significant correlation between gender of the subject and diastolic dysfunction in our study. Due to limitation of number of studies on gender prevalence of diastolic dysfunction in diabetic patients comparison is not possible. 
We found no significant association between different age groups and diastolic dysfunction similar to Kumar VS7 et al who conducted study on 50 patients of DM in Andhra Pradesh in 2017, Patil VC et al ${ }^{10}$ who conducted study on 127 diabetic subjects 2011 in Maharashtra. The maximum number of patients with diastolic dysfunction in our study was in the age group of 50 - 59 years similar to R Madhumati et al ${ }^{11}$ who conducted study on 50 diabetic patients in 2012. There is a significant association of duration of diabetes with diastolic dysfunction similar to Ojji D et al ${ }^{12}$ who studied 120 patients with type 2 diabetes, Patil VC et $\mathrm{al}^{10}$ who conducted study on 127 diabetic subjects in 2011 in Maharashtra, R Madhumati et $\mathrm{al}^{11}$ who conducted study on 50 diabetic patients in 2012 .

There is significant association between mean Hba1c and diastolic dysfunction in our study, similar to Chaudhary AK et al ${ }^{13}$ who studied 100 patients with type 1 diabetes and found out that mean Hba1c was higher in patients with diastolic dysfunction, Patil VC et al ${ }^{10}$ who conducted 127 diabetic subjects 2011 in Maharashtra, and Yadava SK et al ${ }^{14}$ who conducted study on 90 patients with type 2 diabetes in Kathmandu.

we found significant association between diabetic retinopathy and diastolic dysfunction in our study similar to Patil VC et al ${ }^{10}$ who conducted study on 127 diabetic subjects in 2011 in Maharashtra, Kumar VS et al $^{7}$ who conducted study on 50 diabetics. Nakai $\mathrm{H}$ et al ${ }^{15}$ who studied 60 asymptomatic diabetics found no significant association between retinopathy and diastolic dysfunction. However, the parameters taken in their study to detect diastolic dysfunction was longitudinal strain, circumferential strain and radial strain which is different from the parameters we have chosen i.e E / A, E / e' and DT.

In our study we found significant association between urine albumin and diastolic dysfunction similar to, Kumar V S et $\mathrm{al}^{7}$ who conducted study on 50 diabetics. However no of studies correlating the above are very less and the sample size in those studies is also less.

\section{Limitations of the Study}

It is cross-sectional study and thus no causal relationships can be inferred. The subjects were not properly matched with the matched controls. The study was conducted with a small sample size in patients of a tertiary hospital hence generalization of the findings to an average person from community is questionable. Coronary angiography was not performed to rule out the presence of silent coronary artery disease not evident in ECG's.

\section{CONCLUSIONS}

Diabetes is an independent risk factor for cardiovascular disease in the form of diastolic dysfunction in the initial stages. The grade of dysfunction was proportional to duration of diabetes, blood sugar levels and mean HbA1c. Diastolic dysfunction correlates with other microvascular complications of diabetes.

Data sharing statement provided by the authors is available with the full text of this article at jemds.com.
Financial or other competing interests: None.

Disclosure forms provided by the authors are available with the full text of this article at jemds.com.

\section{REFERENCES}

[1] Wild S, Roglic G, Green A, et al. Global prevalence of diabetes: estimates for the year 2000 and projection for 2030. Diabetes Care 2004;27(5):1047-53.

[2] Marcinkiewicz A, Ostrowski S, Drzewoski J. Can the onset of heart failure be delayed by treating diabetic cardiomyopathy? Diabetol Metab Syndr 2017;9:21.

[3] Nichols GA, Gullion CM, Koro CE, et al. The incidence of congestive heart failure in type 2 diabetes. Diabetes Care 2004;27(8):1879-84

[4] Muralidaran Y, Viswanathan P. Diabetic cardiomyopathy: a new perspective of mechanistic approach. J Diabetes Metab 2015;6:10.

[5] Redfield MM, Jacobsen SJ, Burnett JC, et al. Burden of systolic and diastolic ventricular dysfunction in the community: appreciating the scope of the heart failure epidemic. JAMA 2003;289(2):194-202.

[6] American Diabetes Association. Classification and diagnosis of diabetes: standards of medical care in diabetes-2020. 2020;43(Suppl 1):S14-31.

[7] Kumar VS, Sreelatha M, Ramesh K, et al. Study of left ventricular diastolic dysfunction in type 2 diabetes mellitus patients. International Journal of Scientific Study 2017;5(4):219-24.

[8] Boyer JK, Thanigaraj S, Schechtman KB, et al. Prevalence of ventricular diastolic dysfunction in asymptomatic, normotensive patients with diabetes mellitus. Am J Cardiol 2004;93(7):870-5.

[9] Poantă L, Fodor D, Albu A. Left ventricular function in patients with uncomplicated well-controlled diabetes mellitus. Med Ultrason 2010;12(3):184-7.

[10] Patil VC, Shah KB, Vasani JD, et al. Diastolic dysfunction in asymptomatic type 2 diabetes mellitus with normal systolic function. J Cardiovasc Dis Res 2011;2(4):213-22.

[11] Madhumathi R, Gowdaiah PK, Dudhwewala A, et al. Echocardiographic evaluation of diastolic dysfunction in asymptomatic type 2 diabetes mellitus patients. Journal of Evolution of Medical and Dental Sciences 2014;3(1):2009.

[12] Ojji D, Parsonage W, Dooris M, et al. Left ventricular diastolic function in normotensive type-2 diabetic subjects. J Natl Med Assoc 2008;100(9):1066-72.

[13] Chaudhary AK, Aneja GK, Shukla S, et al. Study on diastolic dysfunction in newly diagnosed type 2 diabetes mellitus and its correlation with glycosylated haemoglobin (HbA1c). J Clin Diagn Res 2015;9(8):0C20-2.

[14] Yadava SK, Dolma N, Lamichhane G, et al. Prevalence of diastolic dysfunction in type 2 diabetes mellitus. Kathmandu Univ Med J 2017;15(59):212-6.

[15] Nakai H, Takeuchi M, Nishikage T, et al. Subclinical left ventricular dysfunction in asymptomatic diabetic patients assessed by two-dimensional speckle tracking echocardiography: correlation with diabetic duration. Eur J Echocardiogr 2009;10(8):926-32. 